\title{
EFETIVIDADE DA LOGÍSTICA EM ORGANIZAÇÕES DE ALIMENTOS E BEBIDAS DO ESTADO DE SÃO PAULO: ANÁLISE DOS PLANOS DE AÇÃO ORGANIZACIONAIS
}

Fábio Luciano Violin, Leonardo Giovane Moreira Gonçalves.

Universidade Estadual Paulista - UNESP, curso de Turismo, Campus Experimental de Rosana, SP. E-mail: violin@rosana.unesp.br

\section{RESUMO \\ A competição entre organizações e setores tem se tornado mais laborioso ano a ano e tal entendimento tem sido estudado inúmeros pesquisadores em todos os continentes. Nesse sentido o objetivo deste estudo foi o de apresentar ao longo do período de 2014 até 2017 os níveis médios de efetividade do composto de distribuição de empresas vinculadas ao setor de alimentos e bebidas em organizações do estado de São Paulo. A metodologia contou com a definição do objeto de análise os planos de ação com apontamentos de frequência de itens que apareceram ao longo dos anos nominados em tais planos especificamente no composto de distribuição. Os resultados apontam que o composto de logística traz em seu cerne a potencialidade de estruturar ou desestruturar uma oferta ao permitir que algo seja entregue aos consumidores com mais ou menos qualidade percebida. \\ Palavras-chave: Marketing, Composto de Marketing, Logística, Turismo, Alimentos e Bebidas. \\ EFFECTIVENESS OF LOGISTICS IN ORGANIZATIONS OF FOOD AND BEVERAGES OF THE STATE OF SÃO PAULO: ANALYSIS OF ORGANIZATIONAL ACTION PLANS}

\begin{abstract}
The competition between organisations and sectors has become more laborious year and year and such understanding has been studied numerous researchers on all continents. In this sense the objective of this study was to present over the period of 2014 until 2017 the average levels of effectiveness of the distribution compound of companies linked to the food and beverage sector in São Paulo state organisations. The methodology counted on the definition of the object of analysis the action plans with frequency notes of items that appeared over the nominated years in such plans specifically in the distribution compound. The results indicate that the logistics compound brings in its core the capability of structuring or destructuring an offer by enabling something to be delivered to consumers with more or less perceived quality.
\end{abstract}

Keywords: Marketing, Marketing Mix, Logistics, Tourism, Food and Beverages

\section{INTRODUÇÃO}

A atividade turística, a exemplo das artes, do esporte, da cultura entre outros compõe a percepção, de modo geral, dos elementos que permitem ganho de qualidade de vida e tal entendimento alterou a forma de busca e acesso à informação bem como trouxe para outro patamar os níveis de exigência dos consumidores (LICKORISH; JENKINS, 2002 In: ENGEL et al., 2000).

Os níveis de competitividade nas mais diversas vertentes do setor de turismo alteraram a forma de entendimento de como agir no mercado, as tomadas de decisão apresentaram-se de modo mais complexo e potencialmente gerador de resultados, sejam negativos ou positivos, em outros termos, não é mais possível gerenciar organizações baseadas na tentativa e erro, considerando os resultados desastrosos que tal postura pode gerar. 
Para melhor entendimento, destaca-se que a Embratur (1992) definiu marketing turístico como "conjunto de técnicas estatísticas, econômicas, sociológicas e psicológicas, utilizada para estudar e conquistar o mercado, mediante lançamento planejado dos produtos".

Krippendorf (1989), por seu turno definiu, como as ações que representam uma adaptação sistemática e coordenada da política das empresas de turismo, tanto privadas como do estado objetivando a satisfação de forma plena das necessidades de determinados grupos de consumidores, e obtendo com isso, um lucro apropriado.

Para Cobra (2005) "[...] o turismo é uma importante fonte de receita e a compreensão das oportunidades de mercado permite direcionar as estratégias de marketing". O Marketing Turístico então se apresenta como ferramenta indispensável para o gerenciamento, especialmente no que concerne a diferenciação das ofertas aos segmentos de interesse (MONTEJANO, 2001, In: DIAS; CASSAR, 2005).

Middleton e Clarke (2002) expressa que os conceitos do composto de Marketing podem ser interpretados como um conjunto coordenado de alavancas operadas com o objetivo de se chegar a um determinado ponto. De forma ilustrativa o autor compara as variáveis com um automóvel que possui acelerador, freio, volante e câmbio. O movimento desses controles deve ser utilizado em consonância com as condições da estrada em constante mudança e em conjunto conforme a necessidade mais ampla ou pontual.

Complementarmente, Beni (2003) apregoa que marketing turístico representa um processo pelo qual as organizações do turismo identificam seus clientes tanto reais quanto potenciais. Malhotra (2001) reforça tal posição ao salientar que o estabelecimento de comunicação com esses segmentos ou nichos, com o objetivo de conhecer e influenciar suas necessidades e desejos é vital nas organizações e Melgar (2001) aponta que estas ações têm como objetivo a formulação e adaptação de produtos e serviços para a obtenção de indicadores positivos de satisfação dos usuários ou turistas.

De forma mais específica às atividades relacionadas ao turismo necessitam cada vez mais de estruturação e direcionamento, especialmente se for considerado que, segundo Cobra (2005) as estratégias de marketing turístico tornaram-se fundamentais para o setor na solidificação das tomadas de decisão envolvidas no processo de gerenciamento das ações de estímulo ou resposta ao mercado consumidor de turismo. Dessa forma, as definições apresentadas evidenciam que o papel do marketing turístico é o de analisar, compreender e propor ações que beneficiem de um lado o cliente através da satisfação de suas necessidades e desejos e de outro a empresa através do cumprimento de seus objetivos estabelecidos previamente (PELIZZER, 2005). Middleton e Clarke (2002) reforçam que um dos elementos ou características a serem observados é o fato da heterogeneidade da demanda por parte dos usuários a qual gera diferenças em termos de expectativa e interpretação da oferta de forma tão diversificada que tornam seu julgamento de adequação ou inadequação sujeito a diferentes interpretações e avaliações, informações corroboradas por outros autores tais como Kuazaqui (2001); Aaker e Joachimsthaler (2009); Liu, Kasteridis e Yen (2013).

O produto turístico entendido como toda oferta dispensada aos consumidores é composto tanto de elementos tangíveis tais como equipamentos, infraestrutura, bens físicos e recursos diversos como também intangíveis ao projetar serviços, propostas de preço, elementos ligados a imagem e ao gerenciamento da atividade que de forma sistêmica atuam em conjunto na busca da satisfação de necessidades e desejos reais ou potenciais dos consumidores (DIAS; CASSAR, 2005).

Originalmente esse elemento foi traduzido no Brasil como Praça, contudo, os conceitos associados a ideia de distribuição e mais modernamente logística ganharam espaço ao contemplarem as ações pertinentes a todo processo desde a concepção do produto ou serviços até sua disponibilização para uso dos consumidores. 
As estratégias de distribuição relacionam-se aos canais utilizados dentro do mercado o qual representa segundo Ferrell e Hartline $(2005$, p. 98) um "[...] sistema de organizações pelo qual um produto, recursos, informações e/ ou propriedades flui de produtores a consumidores" e ainda segundo os mesmos autores esse conceito se estende as ações vinculadas à distribuição física a qual foi conceituada como toda a "[...] movimentação de produtos ao local adequado, nas quantidades e no tempo corretos, de maneira eficiente em termos de custo".

Salienta-se que as atividades de distribuição encerram as questões vinculadas, por exemplo, a estocagem movimentação de matérias-primas e materiais, tipos e formas de transporte, organização e uso de equipamentos entre diversos outras ações ou instrumentalizações associadas que sejam necessárias para o correto fluxo de trocas entre os "produtores" e os usuários de produtos, serviços, ideias entre outros.

Segundo Churchill e Peter (2005), o canal de distribuição representa uma rede (ou sistema) organizado de órgãos e instituições que executam todas as funções necessárias para ligar os produtores aos usuários finais.

As estratégias vinculadas ao composto de distribuição analisadas nesse estudo são as seguintes:

Canais: representa o caminho percorrido pelo produto na transferência de quem o produz até quem o consome podendo ser direto ou indireto. Os canais podem ser físicos ou virtuais e incluem atacadistas, varejistas, representantes diretos, vendas diretas ou ainda a conjunção de mais de um ou vários elementos constituintes do canal (KOTLER; ARMSTRONG, 2007).

Cobertura: representa o espaço geográfico coberto pela organização, valendo-se modernamente do conceito de cobertura mundial ou nacional proporcionado pelos meios virtuais (FERRELL; HARTLINE, 2005).

Variedade: refere-se ao conjunto de diferentes possibilidades pelos quais os produtos ou serviços de uma organização podem ser combinados (CHURCHILL; PETER, 2005).

Locais: referem-se ao (s) ponto (s) de distribuição que a organização disponibiliza, bem como a natureza desses locais como forma de analisar de modo correto o tipo, a quantidade e a adequação do que está sendo ofertado ao mercado (KOTLER; KELLER, 2006).

Transporte: refere-se aos meios pelos quais os produtos serão deslocados. O uso de um ou mais tipos de transportes variam conforme a localização geográfica, natureza do bem entre outros elementos. Os meios mais comuns são os transportes rodoviários, ferroviários, aéreos e viários (LAS CASAS, 2007).

O objetivo deste estudo foi o de apresentar os níveis médios de efetividade do composto de logística de empresas vinculadas ao setor de alimentos e bebidas em organizações do estado de São Paulo.

\section{METODOLOGIA}

Para a montagem da base teórica do estudo foram utilizadas fontes secundárias como livros e artigos de natureza científica.

O público alvo foram organizações que tem no setor de Alimentos e Bebidas sua estrutura de oferta mercadológica. Partindo dessa prerrogativa, categorizou-se quatro tipos de empresas: as que atuam em shopping center; as que atuam juntas aos meios de hospedagem (especificamente, restaurantes de hotéis); as que se encontram em unidades conjugadas a postos de combustíveis ou de parada e as que se encontram como oferta isolada em rodovias ou estradas de acesso; e por fim, as convencionais, ou seja, aquelas que se encontram nas ruas e avenidas das cidades.

A amostra manteve-se no estado de São Paulo e atingiu vinte e três cidades (ordem alfabética): Americana; Araraquara; Assis; Bauru; Botucatu; Campinas; Franca; Guaratinguetá; Jaú; Jundiaí; Marília; Mogi das Cruzes; Ourinhos; Piracicaba; Presidente Prudente; Rio claro; Rosana; São Bernardo do Campo; São Carlos; São José do Rio Preto; São Paulo; São Pedro e Sorocaba. 
O objeto de interesse e atuação foram os planos de ação ou documentos equivalentes que demonstrassem a forma de atuação mercadológica das organizações ao longo do período de 2014 até 2017. A participação foi condicionada aos seguintes requisitos: ter um plano de ação ou documento que indicasse a forma de atuação da organização ao longo do período de interesse (2014 -2017) no aspecto mercadológico; que se enquadrasse em uma das categorias destacadas no próximo parágrafo e que disponibilizasse tal material de modo irrestrito para a aferição da efetividade das ações de cada elemento do composto de Marketing em cada uma das vertentes do setor de Alimentos e Bebidas contempladas no estudo.

Após 347 contatos, foi possível obter dados de 36 organizações que atuam em shopping center, 29 restaurantes de hotéis, 22 empresas que encontram em rodovias e estradas de acesso e 28 restaurantes nominados como convencionais. Desse modo, a amostra fica caracterizada como por conveniência configurando acessoriamente sua natureza qualitativa, apesar da apresentação de dados estatísticos.

O levantamento e análise se deu a partir dos itens marca, qualidade, estilo, design, garantias, variedade da oferta e características do produto expressa no texto com a observação da frequência e tipo de discurso recorrente expressos ao longo do período nos planos de ação ou documentos equivalentes.

\section{RESULTADOS}

O elemento do composto de Marketing analisados estão presentes na figura decorrente (figura 1). A figura aponta para o tracionamento que a natureza de cada organização as impele a manter em maior grau de proficiência.

Observa-se que, nesse elemento, os itens são adaptados para inserirem a ideia de como a organização observa o modo de fazer com que seus produtos e serviços sejam processados até o consumo efetivo pelo cliente.

Figura 1: Efetividade logística

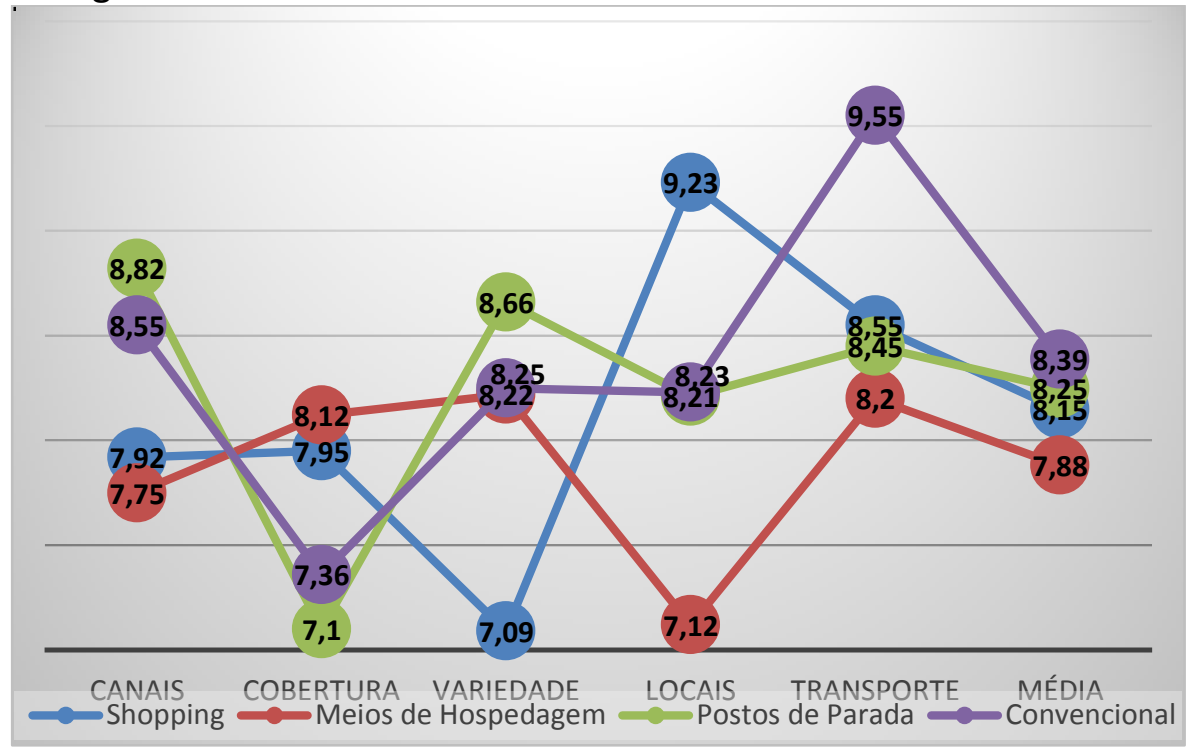

Nesse sentido, boa parte das organizações precisam valer-se de serviços de terceiros para deslocamento de suas matérias primas para confeç̧ão de alimentos e a desatenção a esse composto levou $67 \%$ das organizações a apresentarem falhas, como, falta de produtos, espera pelo não processamento correto ou em tempo de alimentos- sobrecarga de pessoal em horário de 
pico com atendimento deficitário entre outros itens que demonstram a importância da correta alocação dos recursos logísticos no plano de ação das organizações.

Os restaurantes convencionais apresentam os melhores indicadores de proficiência nesse item, porém, as marcas que comercializam seus produtos em shoppings centers apresentam melhor programação do deslocamento, processamento e oferta.

Os restaurantes dos postos de parada têm a maior proficiência na variedade de suas ofertas bem como na utilização correta dos canais de distribuição, considerando sua estabelecida forma de atuação. Por seu tipo de especialidade esse tipo de organização apresenta proficiência inferior média apenas em relação as ofertas realizadas por organizações convencionais.

\section{DISCUSSÃO}

Observa-se que os planos de ação ao longo do período aferido indicam que ao contrário do senso comum, ao menos para o composto de logística aferido dentro do grupo alvo, os meios de alimentação ofertados em shoppings centers apresentam, na média menor proficiência quando comparados com as ofertas dos restaurantes nominados nesse trabalho como convencionais e também os postos de parada.

O item que apresentou menor média geral foi o de cobertura, talvez, justificado pela natureza das organizações as quais trazem itens compulsórios ou próprios de sua natureza ou da sua oferta, isso significa que de certo modo são efetivas ao não fugirem da sua natureza, por exemplo, os restaurantes localizados em shopping center ofertam em geral comida expressa ou com rapidez de acesso, os localizados em meios de hospedagem apresentam especialidade no cardápio, por seu turno os convencionais mantém coesão ao longo do tempo na sua oferta tendo alguns pratos como tradicionais.

\section{CONCLUSÃO}

Destaca-se que o composto de logística tradicionalmente é um dos mais delicados de se trabalhar considerando seu impacto e complexidade.

Tal ponto aparece nos planos de negócios ou documentos similares através da expressa preocupação com a descrição dos itens a serem trabalhados ao longo dos anos analisados. Observa-se que o refinamento desse item encontra eco na busca pela preferência do consumidor especialmente considerando que as atividades logísticas envolvem não apenas a disponibilização dos produtos processados e dos serviços inerentes, mas, a aquisição de produtos da comunidade local, por exemplo, ou ainda a forma de acesso a matérias primas locais ou mesmo exóticas.

Desse modo, observa-se que o senso comum indicaria que as ofertas em shopping center seriam as mais refinadas, não que não sejam, mas algumas de suas características imprimem aos consumidores determinados condicionantes que formatam o tipo de consumidor.

Por seu turno, ao longo do período analisado observa-se que nos últimos dois anos, possivelmente impulsionados pela crise econômica, a preocupação com a redução do retrabalho, desperdício e a otimização de tempo, dinheiro e matéria prima estão mais evidentes.

O composto de logística traz em seu cerne a potencialidade de estruturar ou desestruturar uma oferta ao permitir que algo seja entregue aos consumidores com mais ou menos qualidade percebida.

REFERÊNCIAS BIBLIOGRÁFICAS

AAKER, D. A.; JOACHIMSTHALER, E. Brand Leadership. New York: The Free Press, 2009.

CHURCHILL, G. A. Jr.; PETER, J. P. Marketing: criando valor para os clientes. São Paulo: Saraiva, 2005. 
BENI, M. C. Globalização do turismo: megatendências do setor e realidade brasileira. São Paulo: Aleph, 2003.

COBRA, M. Marketing de Turismo: Turismo, Lazer e Negócios. São Paulo: Cobra, 2005.

DIAS, R.; CASSAR, M. Fundamentos do Marketing Turístico. São Paulo: Prentice Hall, 2005.

EMBRATUR. Decreto 448 de 14 de fevereiro de 1992.

ENGEL, J. F.; BLACKWELL, R. D.; MINIARD, P.W. Consumer Behavior. 10th. Ed. Chicago: Dryden Press, 2000.

FERRELL, O. C.; HARTLINE, M. D. Estratégia de Marketing. São Paulo: Pioneira Thomson Learning, 2005.

KOTLER, P.; KELLER, K. L. Administração de Marketing: A Bíblia do Marketing. 12e ed. Prentice Hall Brasil, 2006.

KOTLER, P.; ARMSTRONG, G. Princípios de Marketing. 12. ed. São Paulo: Prentice Hall, 2007.

KRIPPENDORF, J. Sociologia do Turismo: para uma nova compreensão do lazer e das viagens. Rio de Janeiro: Civilização Brasileira, 1989.

KUAZAQUI, E. Marketing Turístico e de Hospitalidade. São Paulo: Makron Books, 2001.

LAS CASAS; A. L. Marketing de serviços. 5. ed. São Paulo: Atlas, 2007.

LICKORISH, L. J.; JENKINS, C. L. Introdução ao turismo. Rio de Janeiro: Campus, 2002.

LIU, M.; KASTERIDIS, P.; YEN, S. T. Breakfast, lunch, and dinner expenditures away from home in the United States. Food Policy, v. 38, no 2, p. 156-164, 2013.

MALHOTRA, N.K. Pesquisa de marketing: uma orientação aplicada. 3ํe. ed. Porto Alegre: Bookman, 2001.

MELGAR, E. Fundamentos de planejamento e marketing em turismo. São Paulo: Contexto, 2001.

MIDDLETON, V; CLARKE, J. Marketing de Turismo: Teoria e Prática. Rio de Janeiro: Campus, 2002.

MONTENEJANO, J. M. Estrutura do Mercado Turístico. 2ํed. São Paulo: Roca, 2001.

PELIZZER, H. A. Turismo de Negócios: Qualidade na Gestão de Viagens Empresariais. São Paulo: Thomson, 2005. 